\title{
A Comparative Study on the Antinociceptive Effects of Phosphodiesterase Inhibitors on Sciatic Nerve Ligation Induced Neuropathic Pain in Mice
}

\author{
Ali Bazi ${ }^{1}$, Zahra Poodineh², Bentolhoda Behdani², Mehrafrooz Rigi' ${ }^{2}$, Hamideh Arezoomandan², Fatemeh \\ Sahragard², Fatemeh Miri², Najla Anvari ${ }^{3}$, Anita Jahanpanah², Kaveh Tabrizian ${ }^{4,5 *}$ \\ ${ }^{1}$ Faculty of Allied Medical Sciences, Zabol University of Medical Sciences, Zabol, Iran \\ ${ }^{2}$ Student Research Committee, Faculty of Pharmacy, Zabol University of Medical Sciences, Zabol, Iran \\ ${ }^{3}$ Student Research Committee, Nursing and Midwifery School, Zabol University of Medical Sciences, Zabol, Iran \\ ${ }^{4}$ Department of Pharmacology and Toxicology, Faculty of Pharmacy, Zabol University of Medical Sciences, Zabol, Iran \\ ${ }^{5}$ Medicinal Plants Research Center, Zabol University of Medical Sciences, Zabol, Iran
}

\section{*Correspondence to}

Kaveh Tabrizian,

Phone: +98-5432232161,

Fax: +98-32232162

Email: k_tabrizian2005@yahoo.com

Received September 26, 2018 Accepted December 30, 2018

Published online December 31, 2018

\begin{abstract}
Introduction: The role of phosphodiesterase (PDE) inhibitors in reducing neuropathic pains is uncertain. In this study, the antinociceptive effects of theophylline, milrinone, and tadalafil were investigated on sciatic nerve ligation-induced neuropathic pain (NP).

Methods: Male mice (25-30 g) were purchased and housed in controlled environmental conditions before and during the experiments. The mice received identical diet and water ad libitum. Two weeks after sciatic nerve ligation, either theophylline $(75 \mathrm{mg} / \mathrm{kg})$, milrinone $(4.5$ $\mathrm{mg} / \mathrm{kg}$ ), or tadalafil (20 mg/kg) was intraperitoneally (IP) injected for either 1, 3, or 7 consecutive days. Antinociceptive effects were evaluated using the hot plate test. Negative controls received time course IP injections of saline $(5 \mathrm{~mL} / \mathrm{kg})$. A single dose of imipramine $(40 \mathrm{mg} / \mathrm{kg}$, ) was intraperitoneally administrated to the mice in the positive control group.

Results: As was found for this study, a single-dose IP injection of either theophylline $(75 \mathrm{mg} /$ $\mathrm{kg})$, milrinone $(4.5 \mathrm{mg} / \mathrm{kg})$, or tadalafil $(20 \mathrm{mg} / \mathrm{kg})$ on day 14 th following sciatic nerve ligation induced significant antinociceptive effects at 30 minutes $(P<0.01), 60$ minutes $(P<0.01)$, and 90 minutes $(P<0.05)$ compared to the control (saline- treated) animals. Accordingly, both 3- (on days 12-14) and 7-day (on days 8- 14) IP injections of tadalafil $(20 \mathrm{mg} / \mathrm{kg})$ induced significant antinociceptive effects at 30 minutes $(P<0.05), 60$ minutes $(P<0.01)$, and 90 minutes $(P<0.01)$ after sciatic nerve ligation compared to the control (saline-treated) animals. However, the 3and 7-day IP injections of theophylline and milrinone did not reveal any significant differences compared to the control group.

Conclusion: Taken together, the results of this study suggested that selective PDE inhibitors that act predominantly on cGMP pathway, may contribute to the management of sciatic nerve ligation-induced pain.

Keywords: Neuropathic pain, Hot plate test, Phosphodiesterase inhibitors, Imipramine, Mice
\end{abstract}

Please cite this article as follows: Bazi $A$, Poodineh Z, Behdani B. A comparative study on the antinociceptive effects of phosphodiesterase inhibitors on sciatic nerve ligation induced neuropathic pain in mice. Int J Basic Sci Med. 2018;3(4):168171. doi:10.15171/ ijbms.2018.29.

\section{Introduction}

Neuropathic pain (NP) as a primary lesion or dysfunction of the nervous system may be a peripheral or central dysfunction. The peripheral nerves, the plexus, dorsal nerve roots, the spinal cord, and brain are the most commonly influenced organs in this model of pain. ${ }^{1}$ The etiology and pathophysiological pathways involved in NP induced by somatosensory lesions in the peripheral or central nervous system
(CNS) are largely unknown. ${ }^{1-3}$ NP is characterized by allodynia, hyperalgesia, and paresthesia. ${ }^{4}$ Marked degeneration of both myelinated A-fibers and unmyelinated C-fibers as a result of chronic nerve constriction mimics some aspects of NP in humans. ${ }^{1}$ The hot plate test is a behavioral model of nociception which is usually used in monitoring the effects of pain-relieving drugs. ${ }^{5}$

Despite multiple studies on the

(c) 2018 The Author(s); Published by Zabol University of Medical Sciences. This is an open-access article distributed under the terms of the Creative Commons Attribution License (http://creativecommons.org/licenses/by/4.0), which permits unrestricted use, distribution, and reproduction in any medium, provided the original work is properly cited. 
neurobiological mechanism of chronic pain, there are few effective therapies for this type of pain. The principal mechanisms are weakly understood and often noncompliant to conventional antinociceptives such as opiates (limited efficacy in this pain model) and nonsteroidal anti-inflammatory drugs. ${ }^{1}$

Phosphodiesterase (PDE) enzymes regulate the levels of secondary messengers viz cAMP and cGMP, within cells. ${ }^{6}$ Eleven isoenzyme families of PDEs have been recognized with a wide range of functions at cellular and molecular levels. These enzymes promote the substantial effects in the pathogenesis of various diseases, especially through modulating cGMP levels. ${ }^{7}$ It has been reported that elevated levels of cGMP are associated with inhibition of tissue hyperalgesia. ${ }^{6,8}$ Considerable body of evidence has assessed the pivotal role of the balance between CAMP and cGMP in the genesis of tissue hyperalgesia. ${ }^{10-12}$

The antinociceptive effects of milrinone as a PDE-4 inhibitor have been reported previously. ${ }^{6}$ Furthermore, it has been reported that theophylline, a nonspecific PDE inhibitor, exerts dose dependent analgesic and antiinflammatory effects. ${ }^{13}$ It has also been demonstrated that tadalafil, as a PDE-5 inhibitor, exhibits antinociceptive and anti-inflammatory activities in animal models of arthritis. ${ }^{14}$ The purpose of this study was to evaluate the antinociceptive effects of milrinone, theophylline, and tadalafil on sciatic nerve ligation-induced NP in mice, and to further determine the possible involved biochemical pathways.

\section{Materials and Methods}

\section{Animals}

This study was carried out in the Department of Pharmacology and Toxicology, Zabol University of Medical Sciences, Zabol, Iran. Male mice (25-30 g) were purchased from the Animal Breeding and Care Center, Department of Pharmacology, Zabol University of Medical Sciences. All the animals were housed in the controlled environmental conditions $\left(25 \pm 2^{\circ} \mathrm{C}\right.$ and $12: 12$ hours light-dark cycle) before and during the experiments. The mice received identical diet and water ad libitum.

\section{Drugs}

Theophylline (Darou Pakhsh C., Iran), milrinone (Sanofi-Aventis, France), Tadalafil (OSveh Co., Iran) and imipramine (Sobhan Co., Iran) were dissolved in saline and injected intraperitoneally. Ketamine (Alfasan, Holland) and xylazine (Alfasan, Holland) were used for surgical anesthesia.

\section{Antinociceptive Studies}

\section{Hot Plate Test}

Pain sensitivity in sciatic nerve ligated mice (a model of NP) was evaluated using the hot plate test. At first, the animals were anesthetized with ketamine $(80 \mathrm{mg} / \mathrm{kg}$ ) and xylazine $(20 \mathrm{mg} / \mathrm{kg})$ and their right sciatic nerve was ligated by a copper wire. Afterward, the animals were intraperitoneally injected with theophylline $(75 \mathrm{mg} / \mathrm{kg})$, milrinone $(4.5 \mathrm{mg} / \mathrm{kg})$, and tadalafil $(20 \mathrm{mg} / \mathrm{kg})$. All the drugs, namely, theophylline, milrinone, and tadalafil, were administrated for either 1,3, or 7 days, respectively. After sciatic nerve ligation (cut-off time was restricted to 45 seconds) within 90 minutes, a duration of 14 days was determined for latency to licking and lifting paws or jumping from the hot plate surface. Negative and positive control animals were IP injected with saline $(5 \mathrm{~mL} / \mathrm{kg})$ and a single dose of imipramine $(40 \mathrm{mg} / \mathrm{kg})$, respectively.

\section{Statistical Analysis}

Statistical tests were conducted using SPSS software version 19.0. One-way analysis of variance (ANOVA) followed by Newman-Keuls multiple comparison posthoc test and independent samples $t$ test were used to compare the groups. $P$ value $<0.05$ was considered as statistically significant.

\section{Results}

Analgesic Effects of Single-Dose IP injection of PDE Inhibitors to Sciatic Nerve Ligated Mice

As shown in Figure 1 (A-D), a single-dose IP injection of theophylline $(75 \mathrm{mg} / \mathrm{kg})$ to the mice on day 14 , following sciatic nerve ligation, induced significant antinociceptive effects at 30 minutes $(* * * P<0.001), 60$ minutes $(* * P<0.01)$, and 90 minutes $(* P<0.05)$ compared to the animals in the control (saline - treated) group. Milrinone
A

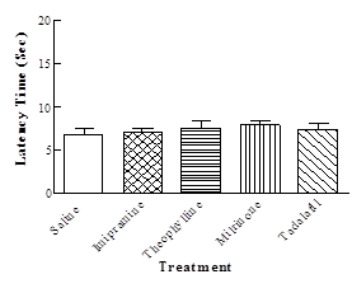

C

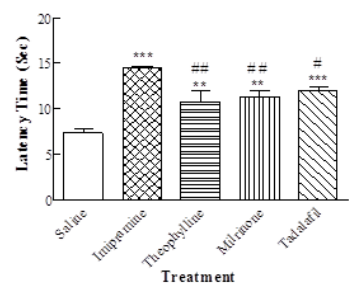

B

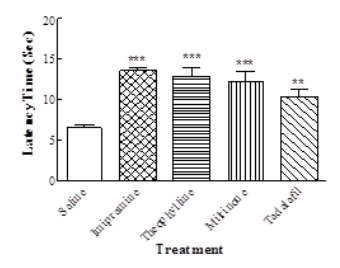

D

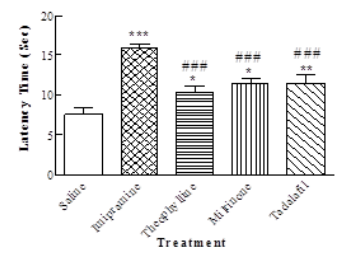

Figure 1. Antinociceptive Effects of Single Dose (Day 14th) IP Injections of PDE Inhibitors, Imipramine (Positive Control), and Saline (Negative Control) to Sciatic Nerve Ligated Mice by Assessing the Latency Response. A, B, C, and D represent assessment of antinociception at 0 (pre-injection), 30, 60, and 90 minutes, respectively, two-weeks after sciatic nerve ligation. Each value represents the mean \pm SEM for 7 mice. Note: $* P<0.05,{ }^{* *} P<0.01$, and ${ }^{* * *} P<0.001$ significantly different from the control (saline-treated) animals. $\# P<0.05$, \#\#P<0.01, and \#\# $\#<0.001$ significantly different from the imipramine - treated animals. 
$(4.5 \mathrm{mg} / \mathrm{kg})$ induced significant antinociceptive effects at 30 minutes $\left({ }^{* * *} P<0.001\right), 60$ minutes $(* * P<0.01)$, and 90 minutes $(* P<0.05)$ after IP injection to the sciatic nerve ligated animals compared to the control (saline-treated) animals. Moreover, in comparison with saline-treated animals (control group), IP injection of tadalafil $(20 \mathrm{mg} / \mathrm{kg})$ to the sciatic nerve ligated animals demonstrated significant antinociceptive effects at 30 minutes $\left({ }^{* *} P<0.01\right), 60$ minutes $(* * * P<0.001)$, and 90 minutes $(* * P<0.01)$. In addition, the analgesic effects of imipramine as a positive control were observed at 30 minutes and persisted until 90 minutes after the IP injection (Figure $1 \mathrm{~B}-\mathrm{D})$.

Antinociceptive Effects of IP Injections of PDE Inhibitors to Sciatic Nerve Ligated Mice in 3-Day and 7-Day Periods As shown in Figure 2 (A-D), IP injection of tadalafil (20 $\mathrm{mg} / \mathrm{kg}$ ) for 3 days (on days 12-14) induced significant antinociceptive effects at 30 minutes $(* * * P<0.001), 60$ minutes $\left({ }^{* *} P<0.01\right)$, and 90 minutes $\left({ }^{* *} P<0.01\right)$ after sciatic nerve ligation compared to the control group (saline-treated animals).

Furthermore, as depicted in Figure 3 (A-D), 7-day IP injections of tadalafil $(20 \mathrm{mg} / \mathrm{kg}$, on days $8-14)$ to the mice under this study induced significant antinociceptive effects at 30 minutes $\left({ }^{*} P<0.05\right), 60$ minutes $(* * * P<0.001)$, and 90 minutes $(* * * P<0.001)$ after sciatic nerve ligation compared to the control animals (saline-treated). However, the 3- and 7-day IP injections of theophylline
A

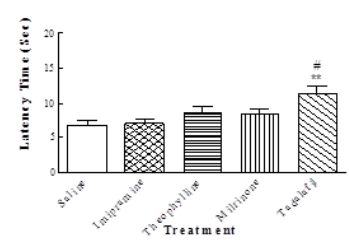

C

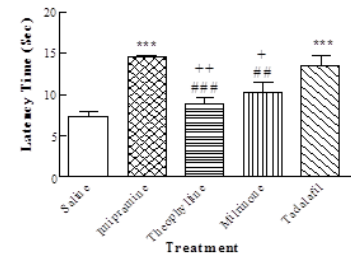

B

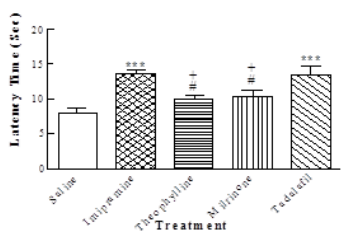

D

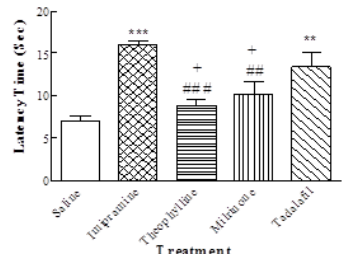

Figure 2. Antinociceptive Effects of 3-Day (days 12th - 14th) IP Injections of PDE Inhibitors, Imipramine (Single Dose), and Saline to Sciatic Nerve Ligated Mice by Assessing the Latency Response. A, B, C, and D represent assessment of antinociception at 0 (pre-injection), 30, 60, and 90 minutes, respectively. Each value represents the mean \pm SEM for 7 mice.

Note: ${ }^{* * P}<0.01$ and ${ }^{* * *} P<0.001$ significantly different from the control (saline - treated) animals. ${ }^{\sharp} P<0.05,{ }^{\sharp \sharp} P<0.01$, and ${ }^{\sharp \#} P<0.001$ significantly different from the imipramine-treated animals. ${ }^{+} P<0.05$ and ${ }^{++} P<0.01$ significantly different from the tadalafil-treated animals. and milrinone did not reveal any significant differences compared to the control animals.

\section{Discussion}

The molecular mechanisms of hyperalgesia mediated by the factors such as prostanoids and sympathomimetic amines have been incompletely addressed. There are suggestions indicating a significant role for the balance of $\mathrm{Ca}^{2+} /$ cAMP and cGMP neuronal content in this process. ${ }^{6}$ In particular, an essential role has been highlighted for cAMP in determining the sensitivity of sensory neurons to hyperalgesic stimulus as evidenced by the action of PDE inhibitors. ${ }^{6}$

The results of the present study indicated that single dose IP injection of theophylline $(75 \mathrm{mg} / \mathrm{kg}$, as a nonselective PDE inhibitor), milrinone ( $4.5 \mathrm{mg} / \mathrm{kg}$, as a PDE4 inhibitor), and tadalafil (20 mg/kg, as a PDE5 inhibitor) induced significant anti-nociceptive effects in the sciatic nerve ligated mice. Findings of this study proved significant differences between all these mentioned PDE inhibitors and imipramine- and saline- treated groups. In addition, both 3- and 7-day IP injections of tadalafil $(20 \mathrm{mg} / \mathrm{kg})$ induced significant antinociceptive effects in comparison with the negative control animals (saline-treated). However, 3- and 7-day IP injections of theophylline and milrinone did not cause any significant differences in comparison with the control animals.

Several bodies of evidence have demonstrated that it is possible to modulate hyperalgesia via adjustment of
C

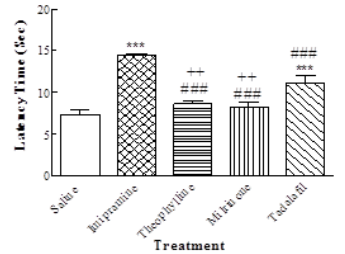

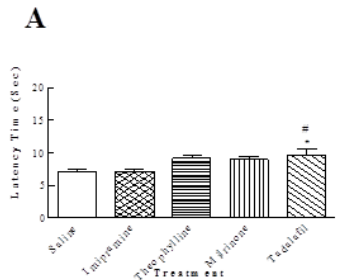

B

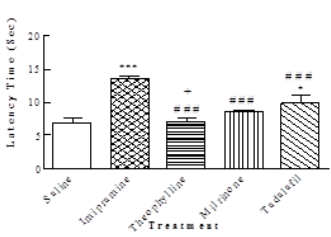

D

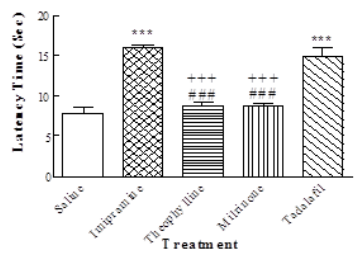

Figure 3. Antinociceptive Effects of 7-Day (Days 8th - 14th) IP Injections of PDE Inhibitors, Imipramine (Single Dose), and Saline to Sciatic Nerve Ligated Mice by Assessing the Latency Response. A, B, C, and $\mathrm{D}$ represent assessment of antinociception at 0 (pre-injection), 30, 60, and 90 minutes, respectively. Each value represents the mean \pm SEM for 7 mice.

Note: $* P<0.05$ and ${ }^{* * *} P<0.001$ significantly different from the control (saline-treated) animals. ${ }^{\sharp} P<0.05$ and ${ }^{\sharp \#} P<0.001$ significantly different from the imipramine - treated animals. ${ }^{+} P<0.05,{ }^{++} P<0.01$, and ${ }^{+++} P<$ 0.001 significantly different from the tadalafil-treated animals. 
the intracellular levels of cAMP or cGMP in peripheral sensitive neurons. ${ }^{8,9,15}$ Studies have shown that elevated levels of cAMP and cGMP are associated with the increase and decrease in the tissue hyperalgesia, respectively. ${ }^{6} \mathrm{~A}$ considerable body of literature has evaluated the pivotal role of the balance between cAMP and cGMP in the biogenesis of hyperalgesia. ${ }^{6,10-12}$ Sildenafil was reported to induce antinociception via the inhibition of cGMP degradation. ${ }^{8}$ Furthermore, the contribution of the NO/cGMP signaling pathway has been mentioned in nociception in previous studies. ${ }^{9}$

\section{Conclusion}

To conclude, the findings of this study showed a relationship between analgesia in sciatic nerve ligated mice as a model of NP and the activation of cGMP pathway. The balance between the intracellular levels of cAMP and cGMP may stand as a possible candidate for developing new peripheral analgesics.

\section{Ethical Approval}

The study protocol was approved by the Local Ethics Committee for Animal Experimentation, Zabol University of Medical Sciences, Zabol, Iran.

\section{Competing Interests}

None.

\section{References}

1. Karimi GHR, Tabrizian K, Rezaee R. Evaluation of the analgesic effects of dextromethorphan and its interaction with nitric oxide on sciatic nerve ligated rats. J Acupunct Meridian Stud. 2010;3(1):38-42. doi: 10.1016/S20052901(10)60006-4.

2. Hashemzaei M, Sadeghi Bonjar MA, Tabrizian K, Iranshahi M, Iranshahy M, Rezaee R. Evaluation of the analgesic effect of Umbelliprenin and Umbelliprenin-morphine co-administration on the acute, chronic and neuropathic pain. Indian J Pharm Educ. 2015;49:121-5. doi: 10.5530/ ijper.49.2.7

3. Miri AH, Sharifi-Rad J, Tabrizian K, Nasiri AA. Antinociceptive and anti-inflammatory activities of Teucrium persicum Bois extract in mice. Scientifica. 2015;2015:ID972827. doi: 10.1155/2015/972827
4. Kaur M, Singh A, Kumar B, et al. Protective effect of coadministration of curcumin and sildenafil in alcohol induced neuropathy in rats. Eur J Pharmacol. 2017;805:5866. doi: 10.1016/j.ejphar.2017.03.012

5. Espejo EF, Mir D. Structure of the rat's behaviour in the hot plate test. Behav Brain Res. 1993;56(2):171-176.

6. Karimfar MH, Gholizadeh Sh, Hajrasouliha AP, et al. Low dose morphine enhances morphine antinociception effects in the animals pretreated with selective and nonselective phosphodiesterase inhibitors. Iran J Pharm Sci. 2009;5(2):73-81.

7. Boswell-Smith V, Spina D, Page CP. Phosphodiesterase inhibitors. Br J Pharmacol. 2006;147(S1):S252-S257. doi: 10.1038/sj.bjp.0706495

8. Jain NK, Patil CS, Singh A, Kulkarni SK. Sildenafil, a phosphodiesterase-5 inhibitor, enhances the antinociceptive effect of morphine. Pharmacology. 2003;67:150-6. doi: 10.1159/000067802

9. Tao YX, Hassan A, Haddad E, Johns RA. Expression and action of cGMP-dependent protein kinase alpha in inflammatory hyperalgesia in rat spinal cord. Neuroscience. 2000;95:525-33. PMID:10658633

10. Durate ID, Lorenzetti BB, Ferreira SH. Peripheral analgesia and activation of the nitric oxide-cGMP pathway. Eur J Pharmacol. 1990;186:289-93.

11. Kress M, Rodl J, Reeh PW. Stable analogues of cAMP but not cGMP sensitize unmyelinated primary afferents in rat skin to heat stimulation but not to inflammatory mediators, in vitro. Neuroscience 1996;74:609-617.

12. Taiwo YO, Levine JD. Further confirmation of the role of adenyl cyclase and of cAMP-dependent protein kinase in primary afferent hyperalgesia. Neuroscience. 1991;44:1315 .

13. Kumar A, Jain NK, Kulkarni SK. Analgesic and antiinflammatory effects of phosphodiesterase inhibitors. Indian J Exp Biol 2000;38(1):26-30.

14. Rocha FA, Silva FS Jr, Leite AC, et al. Tadalafil analgesia in experimental arthritis involves suppression of intraarticular TNF release. Br J Pharmacol. 2011;164(2b):828835. doi:10.1111/j.1476-5381.2011.01469.x.

15. Mamiya T, Noda Y, Ren X, et al. Involvement of cAMP systems in morphine physical dependence in mice: Prevention of development of morphine dependence by rolipram, a phosphodiesterase 4 inhibitor. Br J Pharmacol. 2001;132(5):1111-1117. doi: 10.1038/sj.bjp.0703912 